\title{
BMJ Open Doctors' approaches to PSA testing and overdiagnosis in primary healthcare: a qualitative study
}

\author{
Kristen Pickles, ${ }^{1}$ Stacy M Carter, ${ }^{1}$ Lucie Rychetnik ${ }^{1,2}$
}

To cite: Pickles K, Carter SM, Rychetnik L. Doctors' approaches to PSA testing and overdiagnosis in primary healthcare: a qualitative study. BMJ Open 2015;5: e006367. doi:10.1136/ bmjopen-2014-006367

- Prepublication history for this paper is available online. To view these files please visit the journal online (http://dx.doi.org/10.1136/ bmjopen-2014-006367).

Received 12 August 2014 Revised 16 February 2015 Accepted 17 February 2015

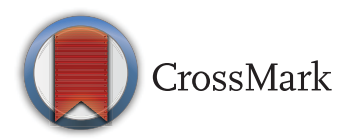

\footnotetext{
${ }^{1}$ Centre for Values, Ethics and the Law in Medicine, Sydney School of Public Health, University of Sydney, Sydney, New South Wales, Australia

${ }^{2}$ School of Medicine, University of Notre Dame, Sydney, New South Wales, Australia
}

\section{Correspondence to}

K Pickles;

kristen.pickles@sydney.edu. $\mathrm{au}$

\section{ABSTRACT}

Objectives: (1) To explain general practitioners' (GPs') approaches to prostate-specific antigen (PSA) testing and overdiagnosis; (2) to explain how GPs reason about their PSA testing routines and (3) to explain how these routines influence GPs' personal experience as clinicians.

Setting: Primary care practices in Australia including men's health clinics and rural practices with variable access to urology services.

Participants: 32 urban and rural GPs within Australia. We included GPs of varying ages, gender (11 female), clinical experience and patient populations. All GPs interested in participating in the study were included.

Primary and secondary outcome measure(s): Data were analysed using grounded theory methods to determine how and why GPs provide (or do not provide) PSA testing to their asymptomatic male patients. Results: We observed patterned variation in GP practice, and identified four heuristics to describe GP preference for, and approaches to, PSA testing and overdiagnosis: (1) GPs who prioritised avoiding underdiagnosis, (2) GPs who weighed underdiagnosis and overdiagnosis case by case, (3) GPs who prioritised avoiding overdiagnosis and (4) GPs who did not engage with overdiagnosis at all. The heuristics guided GPs' Routine Practice (usual testing, communication and responses to patient request). The heuristics also reflected GPs' different Practice Rationales (drawing on experience, medicolegal obligations, guidelines and evidence) and produced different Practice Outcomes (GPs' experiences of the consequences of their PSA testing decisions). Some of these heuristics were more responsive to patient preferences than others.

Conclusions: Variation in GPs' PSA testing practices is strongly related to their approach to overdiagnosis and underdiagnosis of prostate cancer. Men receive very different care depending on their GP's reasoning and practice preferences. Future policy to address overdiagnosis will be more likely to succeed if it responds to these patterned variations.

\section{INTRODUCTION}

Prostate-specific antigen (PSA) testing for prostate cancer in healthy men is an emotive, controversial ${ }^{1}$ and hotly debated

\section{Strengths and limitations of this study}

Most previous research has examined which general practitioner (GP) characteristics are associated with frequent or infrequent testing, and has been predominantly quantitative. This in-depth qualitative study offers a unique examination of GPs' approaches to prostate cancer overdiagnosis, from the GP perspective. It is the first study to systematically map prostate-specific antigen (PSA) testing with GPs' reasoning and understanding of prostate cancer screening and overdiagnosis.

- We identified and interviewed highly informative participants (GPs) and have developed valuable detailed insights into how the PSA testing process works in everyday practice.

- There may be value in knowing the prevalence of the four heuristics we have discovered across the population: a next step could be a populationbased survey to ascertain the prevalence of these heuristics at a population level.

- Since physicians with strong opinions may have been more likely to volunteer, some selection bias is possible. However, diverse opinions and approaches were reported, suggesting that strong selection bias is unlikely.

issue. Evidence suggests that harms of PSA testing for prostate cancer in asymptomatic men can outweigh benefits. ${ }^{2-4}$ Most guidelines recommend against population screening; ${ }^{35-7}$ however, some professional societies do recommend selective PSA testing ${ }^{8-10}$ (table 1). In Australia, and internationally, many men continue to be tested despite guidelines advising not to screen. ${ }^{16-18}$ This article presents an empirical qualitative study of how Australian general practitioners (GPs) reason about PSA testing of asymptomatic men for prostate cancer, who they test and why, with a particular focus on how GPs manage the risk of overdiagnosis.

Overdiagnosis and/or overtreatment are considered the main potential harms of PSA testing. Overdiagnosis occurs when people without symptoms are correctly diagnosed 
Table 1 The US, UK and Australian recommendations for prostate-specific antigen (PSA) testing of asymptomatic men for prostate cancer

Professional body

Population US Preventive Services Task Force (USPSTF) $^{3}$

National Health and Medical Research Council (NHMRC) ${ }^{11}$ National Health Service (NHS) ${ }^{12}$

National American Cancer Society $(\mathrm{ACS})^{10}$

Cancer Council Australia (CCA) and Australian Health Ministers' Advisory Council (AHMAC), 2010*13

Specialist American Urological Association $(\mathrm{AUA})^{8}$

Urological Society of Australia and New Zealand (USANZ) ${ }^{14}$

Primary American College of Physicians

Care $(\mathrm{ACP})^{15}$

Royal Australian College of General Practitioners (RACGP) 6
Advice for health practitioners (see original documents for exact phrasing)

- Discuss PSA screening thoroughly with men who raise the issue or if the man's individual circumstances warrant consideration of PSA testing. Do not feel obligated to offer PSA testing if a patient does not raise the issue or request the test

- The decision to start or continue PSA screening should reflect the patient's understanding of the possible benefits and expected harms and should respect his preferences

- Before ordering a PSA test, health practitioners should talk to men about the potential benefits and harms of PSA testing

- Screening not recommended. An informed choice programme, Prostate Cancer Risk Management aims to provide high-quality information about the risks and benefits to men who ask about screening in order to enable them to decide whether to have the test

- Provide men the opportunity to make an informed decision; for men who are unable to decide, the screening decision can be left to the discretion of the healthcare provider

- Men at average risk and expected to live at least 10 more years should receive this information beginning at age 50 years. Men in higher risk groups should receive this information at age 4045 years

- Speak to men about the benefits and harms of testing and treatment so that they can make an informed choice

- Shared decision-making for men aged 55-69 years based on a man's values and preferences

- Routine screening is not recommended in men aged 40-54 years at average risk, or in men over 70 years or with less than a 10-15-year life expectancy; decisions should be individualised for men younger than 55 years at higher risk

- PSA and digital rectal examination (DRE) should be offered to men 55-69 years, after providing information about the risks and benefits of such testing

- Interested men in younger age groups (under 55 years) could have a single PSA test and DRE performed at or beyond age 40 to provide an estimate of their prostate cancer risk over the next 10-20 years, with the intensity of subsequent monitoring being individualised accordingly

- Inform men 50-69 years about the limited potential benefits and substantial harms of screening for prostate cancer

- Base the decision on the man's risk for prostate cancer, a discussion of the benefits and harms of screening, the patient's general health and life expectancy and patient preferences

- Advised not to screen patients who do not express a clear preference for screening

- Advised not to screen average-risk men under 50 years, over 69 years, or with a life expectancy of less than 10 to 15 years

- Not recommended unless the man specifically asks for it, and he is fully counselled on the pros and cons

- General practitioners need not raise this issue, but if men ask about prostate screening they need to be fully informed of the potential benefits, risks and uncertainties of prostate cancer testing

- When a patient chooses screening, both PSA and DRE should be performed

- Responding to the patient's concerns and fulfilling medicolegal responsibilities are considerations in discussion with patients 
with a disease that would not cause them to experience symptoms or early death. ${ }^{19}$ It is hard to understand and explain, ${ }^{20}$ and difficult to quantify; estimates range from $15 \%$ to more than $84 \%$ of screen-detected prostate cancers. ${ }^{21-26}$ Overdiagnosis may lead to overtreatment: ${ }^{27}$ treatment a person did not need. PSA testing often triggers a cascade of diagnostic tests and active treatment, ${ }^{28} 29$ potentially compromising a well person's quality of life. ${ }^{30}$ Advocates of testing argue that PSA testing may, in some cases, lower the stage and grade of cancer at diagnosis, and reduce the risk of being diagnosed with metastatic prostate cancer, for which there is no cure.$^{32-34}$ However, across the population of asymptomatic men, PSA testing does not decrease all-cause mortality, and some men will progress and develop metastatic disease even if they are screened (despite earlier diagnosis). ${ }^{35}$

Responsibility for guiding men's decisions about whether or not to be screened for prostate cancer has largely been placed in the hands of individual physicians. In Australia, GPs are the primary point of contact to access a PSA test. There is no organised screening programme; PSA testing is opportunistic but prevalent. ${ }^{18}$

Empirical work exploring prostate cancer screening in general practice has primarily focused on: (1) the reasons GPs give for ordering PSA tests; (2) the characteristics of GPs (such as age, gender, location) associated with more or less frequent testing and (3) how GPs communicate with patients about the PSA test. $^{36-44}$ The predominantly quantitative evidence provides insights into the patterns and potential drivers of PSA testing in general practice but does not illuminate the dilemmas of PSA testing from the GP's perspective, and in particular how GPs reason about overdiagnosis. To fill this gap, we conducted a qualitative study to explore how and why GPs provide (or do not provide) PSA testing to their asymptomatic male patients. We report on the significance and impact of overdiagnosis in GPs' clinical reasoning about PSA testing.

\section{METHODS}

\section{Design}

We used the well-established, systematic qualitative research methodology of grounded theory ${ }^{45}$ to guide our sampling and analysis. We collected data via in-depth interviews. GPs had an opportunity to discuss the study, and gave consent prior to participation.

\section{Participants and setting}

We recruited 32 urban and rural GPs throughout Australia (11 female). Our initial purposive sample was of GPs working in men's health clinics in Sydney $(n=2)$. We advertised via the newsletters and email lists of regional GP organisations (Medicare Locals) in Sydney $(n=8)$. GPs were invited to contact KP if they were interested and willing to participate in the research. We then broadened our sampling by advertising in mass and social media, and in medical journals (Medical Observer, the Australian Medical Association's GP Network News, and the $6 \mathrm{~min}$ newsletter). As analysis and sampling evolved, we invited additional rural and interstate GPs to answer specific analytical questions $(n=11)$. Rural GPs were accessed by phoning practice managers, through colleagues, and advertising with rural Medicare Locals, adding eight further GPs. When we encountered GPs whose routine care was divergent from previously interviewed GP norms, we invited more GPs from that practice to attempt to distinguish between personal and institutional influences on their practice. An additional three GPs were recruited in this final phase of theoretical sampling. GPs of varying ages, clinical experience, gender and patient populations were all included. All GPs interested in participating in the study were included. GPs were compensated for their time.

\section{Interviews/data collection}

A semistructured interview schedule was developed with a focus on GPs' current approaches to, and reasoning about, PSA testing. The schedule covered a broad range of topics, including GPs' recent clinical encounters involving PSA testing decisions; communicating information; screening pathways; and the central theme of this paper, overdiagnosis. The interview schedule was modified between interviews, informed by the developing analysis. Interviews took place between March and September 2013. They were conducted by KP, mostly by telephone, and ranged in duration from $18 \mathrm{~min}$ to $1 \mathrm{~h}$ and $10 \mathrm{~min}$. All interviews were audio-recorded, de-identified and transcribed verbatim.

Examples of questions GPs were asked about overdiagnosis included the following:

- Are you familiar with the term 'overdiagnosis'?

- Do you think about the issue of overdiagnosis in your practice?

- How do you manage overdiagnosis in your practice?

- Overdiagnosis must be a challenging concept to talk about with your patients; how do you manage that challenge?

\section{Data coding and analysis}

The analysis was led by KP, who coded the transcripts and wrote detailed memos which were reviewed and discussed by the authors in analytical meetings. A subset of transcripts was read and coded by all three authors independently; this coding was compared and discussed to inform the development of the central concepts in the study. This paper focuses on how GPs dealt with the concept of overdiagnosis.

\section{RESULTS}

Most GPs felt uncertain and/or conflicted regarding what to do about PSA testing of asymptomatic men. 
In the following section, we will explain overall patterns and then outline four heuristics used in practice.

\section{GPs considered underdiagnosis as well as overdiagnosis}

GPs discussed the harms of underdiagnosis (the missed opportunity to intervene in potentially preventable deaths) as much as those of overdiagnosis (the psychological and physical harms and financial costs of unnecessary diagnosis and treatment). Since both harms are salient and serious, PSA testing decisions were described as a "balancing act" (GP21) or gamble. GPs reported the difficulties of needing to choose between potential harms (eg, incontinence and impotence) and the chance of saving lives.

\section{Testing decisions were described as a personal burden}

Uncertainty about PSA testing created a 'personal burden' for some GPs; they felt personally responsible for the consequences of their PSA testing approach, and experienced guilt and self-blame as a result.

Many GPs used personal or professional experiences with the PSA test, both positive and negative, as powerful anchors for their current practice: these experiences often explained GPs' perception of personal burden. We will return to the personal burden of PSA testing throughout the following sections.

\section{GPs' communication practices varied}

GPs varied in the conversations they had with men specifically about overdiagnosis. Some deliberately avoided raising the issue, or talked men into or out of having a PSA test. GPs described several important contextualising factors.

1. Cancer is widely feared and difficult to talk about.

2. Overdiagnosis is hard to understand for GPs and for the public-and it is contradictory to many people's existing health beliefs.

3. Both doctors and patients often have a strong belief that cancer screening is, in general, a worthwhile and important strategy to combat the risk of getting cancer.

\section{GPs employed four heuristics to manage PSA testing}

GPs' responses to this difficult situation depended on how they viewed an implicit continuum between overdiagnosis and underdiagnosis. They considered which end of the spectrum would cause the greatest harm to each patient and/or their patients in general.

Four broad patterns ('heuristics') were employed.

1. Some GPs preferred to offer PSA testing to avoid underdiagnosis.

2. Some GPs were strongly oriented to avoiding overdiagnosis, and so tried to test as little as possible.

3. Some GPs made case-by-case individualised decisions.

4. Some GPs did not think about underdiagnosis or overdiagnosis at all.

These four heuristics represent observed patterns of GPs' preferred or dominant practice orientations; that is, each
GP seemed to prefer one of these four heuristics as their overall approach to PSA testing. Some of these heuristics were more responsive to patient preferences than others (table 2).

The GPs' Dominant Practice Orientation guided their Routine Practice (usual testing, communication and responses to patient requests). GPs also described their Practice Rationale (drawing on experience, medicolegal obligations, guidelines and evidence) which influenced testing decisions and justified why they adopted their particular practice orientation. Their orientation produced a Practice Outcome: GPs' experiences of the consequences of their PSA testing decisions. The four dominant practice orientations (heuristics) are summarised in table 2 and described below.

\section{Heuristic 1: GP preference to offer PSA testing to avoid underdiagnosis}

GPs employing heuristic 1 thought testing was necessary because there was a possibility it might prevent a man's death. Overdiagnosis was perceived as (1) a natural consequence of PSA testing; (2) better than dying and (3) a justifiable source of harm (harms being a regrettable but necessary price of 'cure')

These GPs focused on cancer as life-threatening, and prostate cancer as a terrible death. They saw preventing death as the primary duty of the GP. This heightened their responsibility to do anything that may diagnose cancer early: "Because if you don't overdiagnose, the alternative is to underdiagnose" (GP28). Underdiagnosis was perceived to be a medicolegal risk, and for some GPs, legal risk was uppermost in their minds during the decision-making process. GPs concerned with missing diagnosing cancers practised more defensively; "I'm often a bit defensive...I guess that's partly that legal thing" (GP5).

GPs with this practice orientation advised men to have a PSA, emphasising benefits of early detection, and did not discuss overdiagnosis.

Some of these GPs thought decisions about postdiagnosis management (eg, active surveillance) could limit the harms of potential overdiagnosis. This allowed them to define testing without invasive procedures as inconsequential: "it's not terribly onerous to have a blood test every six months" (GP3). Although many of these GPs accepted that the PSA test was not perfect, they preferred to test because "clearly, people's lives are saved" (GP8)

These GPs anchored their practice orientation to personal experiences. Their approach was supported by stories of men still being alive following active testing and treatment.

Another anchor for this heuristic was having experienced caring for patients with metastatic cancers, "I've had two recently where their GP refused to actually test the PSA level over the last ten years and both presented with metastatic prostate cancer" (GP24), and witnessing the horrors of prostate cancer deaths: "dying from prostate cancer would probably rank amongst one of the 


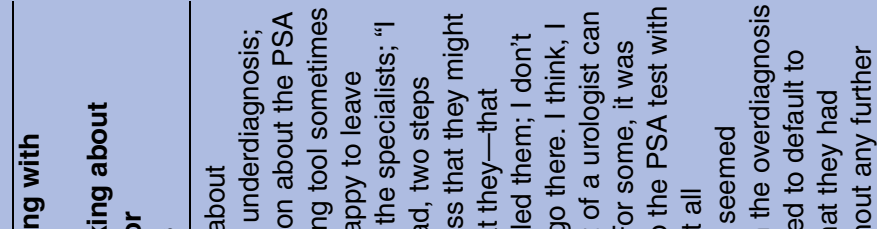

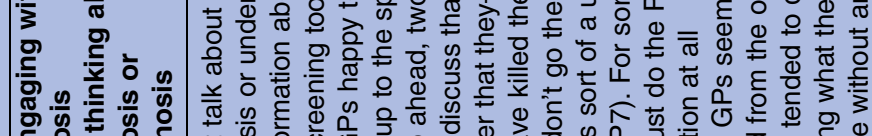

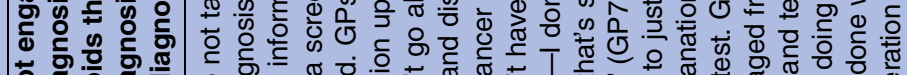

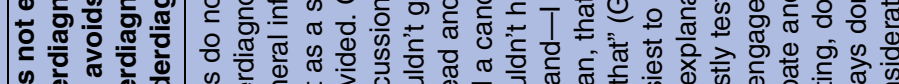

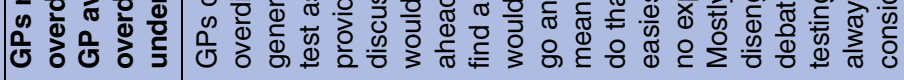

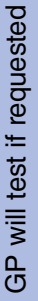

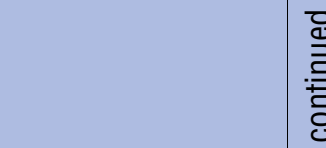

얼

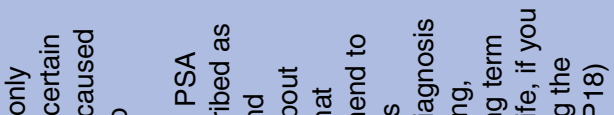

里罾

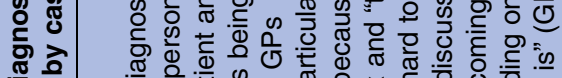

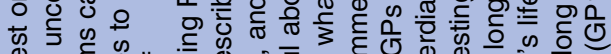
ఖ

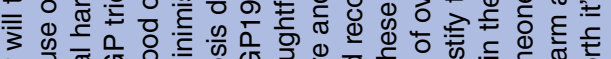
了。 ه্ o

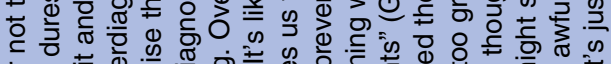

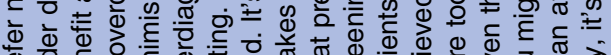

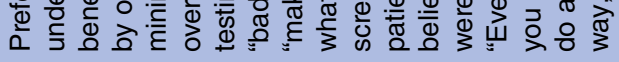

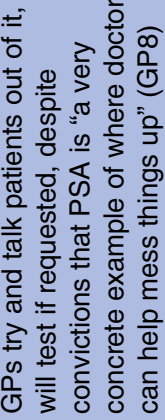

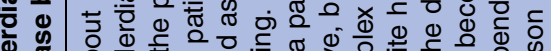

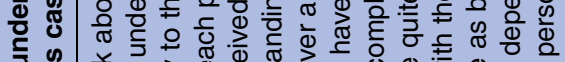

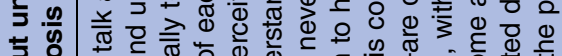

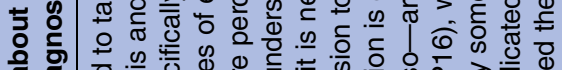

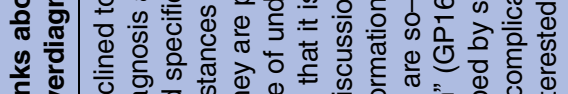

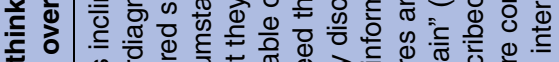

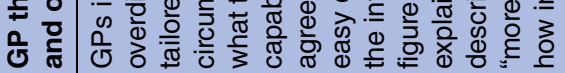

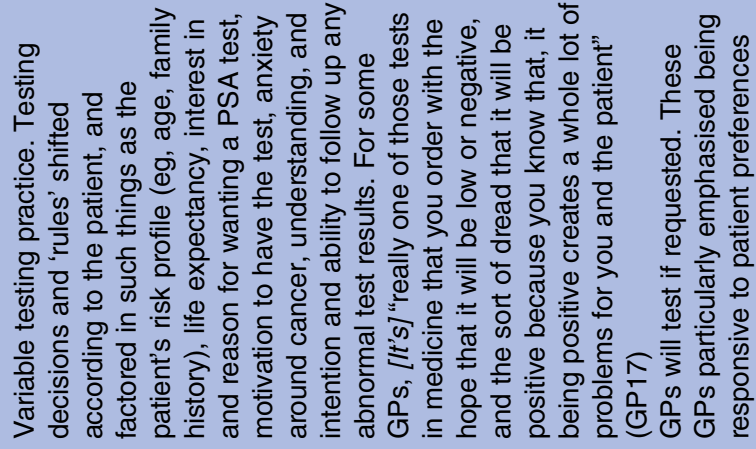

공 을 = $\stackrel{0}{\bar{\varpi}}$

ब。

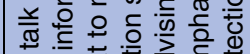

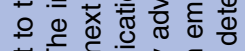

\%

\&

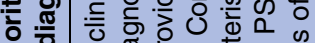

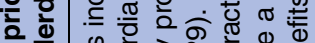

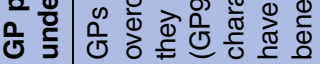

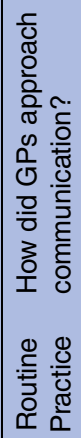

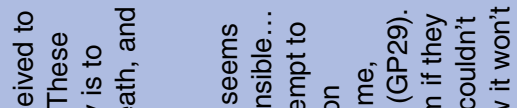

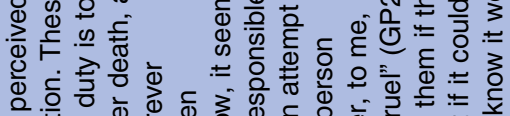

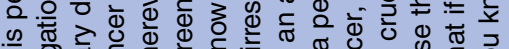

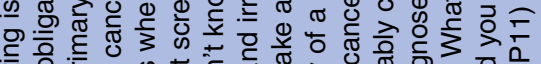
के

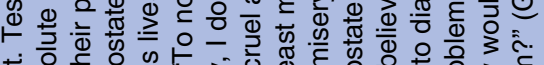

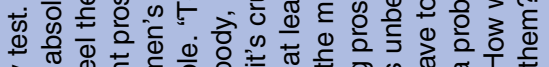

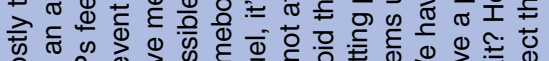

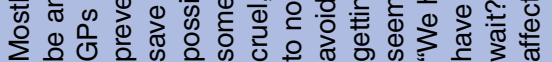

윰

음

के

응

초임

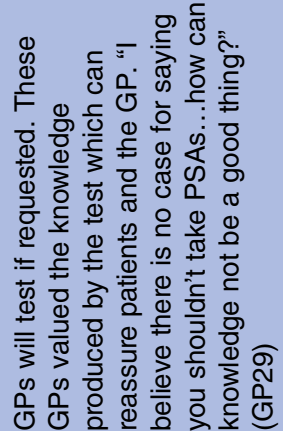

유

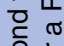

oㅇํㅇ

党需

웧ㅇㅇ

음

오 옹 


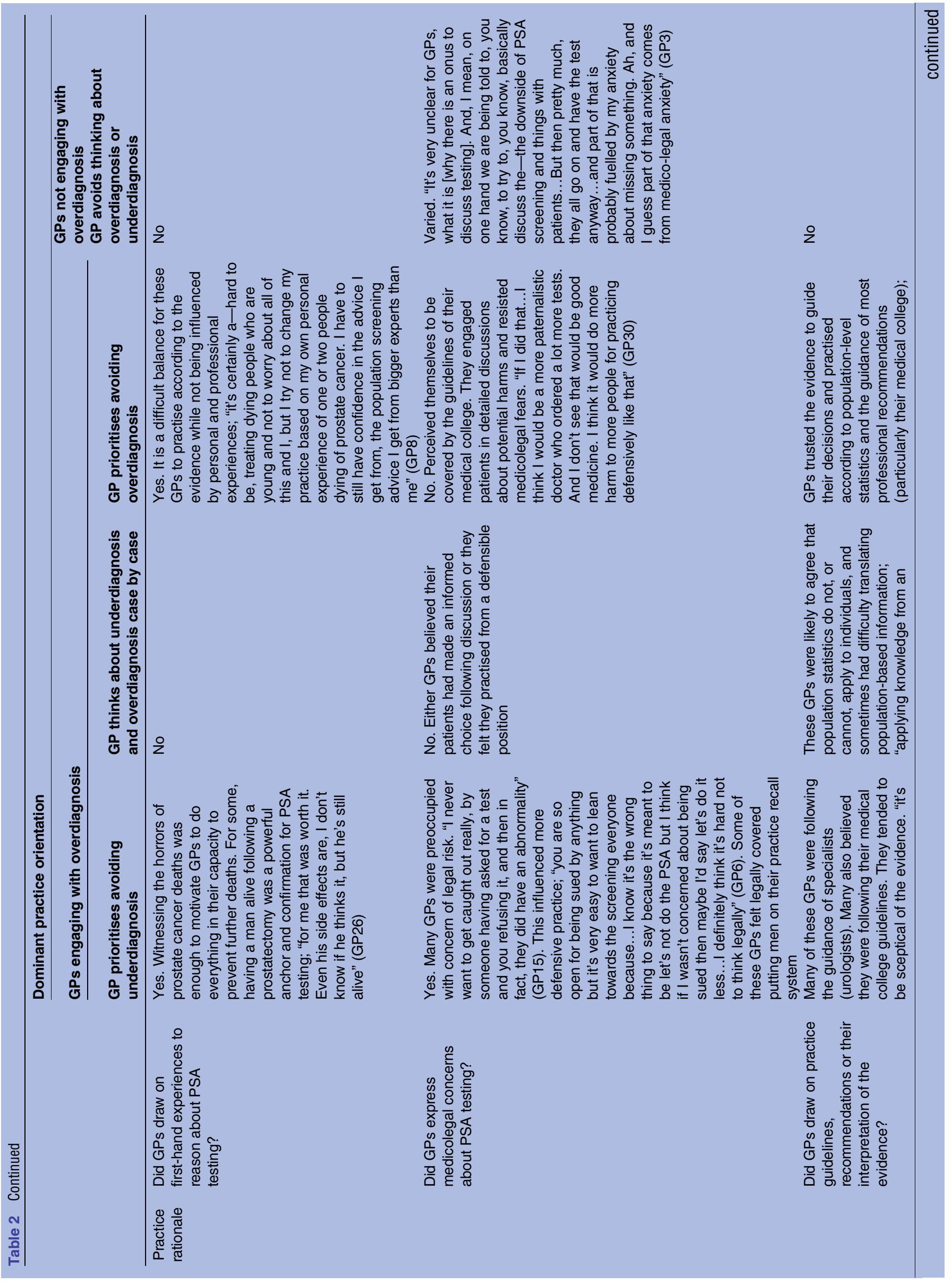




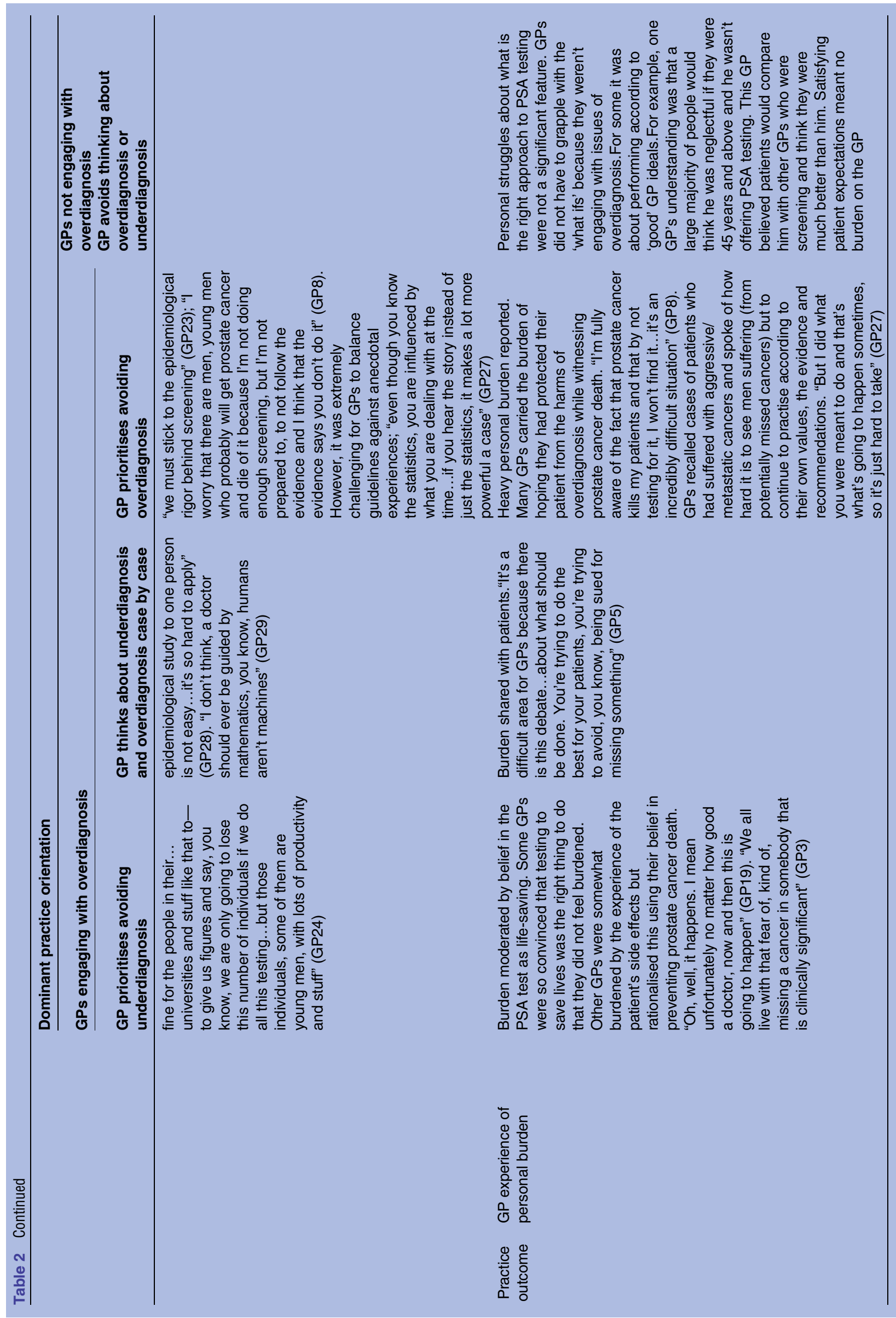


worst ways to die...pain that is almost not able to be alleviated by narcotics" (GP29).

Despite their convictions, GPs experienced some personal struggle when they witnessed side effects of prostate cancer treatment. Such cases were often recalled in detail. For example, one GP describes this as "a heavy burden if a person is left with side effects" but accepted that "that's just part of being a GP, you have to walk around with this" (GP29). They tried not to take it personally, "oh, well, it happens. I mean, unfortunately, no matter how good a doctor [I am], now and then this is going to happen" (GP19). Overall, though, the personal burden felt by this group was relatively small and did not challenge the GP's belief in PSA testing, which they said fulfilled their role as a clinician to save lives. Many also regarded testing as consistent with specialists' advice, which allowed them to reduce their personal burden; that is, the responsibility of decisions about PSA testing was shared with these specialists (but not with their patients).

\section{Heuristic 2: GP preference to not offer PSA testing to avoid overdiagnosis}

GPs employing heuristic 2 preferred not to conduct PSA testing. Their primary justification was preventing harms caused by overdiagnosis. However, while they would try to talk patients out of having the test, they would never refuse a PSA test. These GPs also recognised that PSA testing has saved lives; "we know that happens. The problem is, it just doesn't happen often enough to balance out...all the damage that we do" (GP17).

This group of GPs emphasised the harms of PSA testing (including overdiagnosis) when advising their patients; and said many patients chose not to be tested following discussion. These GPs, who fully explain overdiagnosis, described themselves as "taking the risk of doing the hard work, hard yards" (GP23). They resisted medicolegal fears by engaging in detailed discussions of benefits and harms, and felt covered from legal prosecution by the Royal Australian College of General Practitioners (RACGP) guidelines.

GPs committed to avoiding overdiagnosis particularly drew on and trusted the research evidence to guide and inform their testing decisions. However, practising accordingly could be compromised by situational factors, such as a patient who had not been tested dying of prostate cancer. GPs said it was incredibly challenging to ignore personal (anecdotal) experiences, yet some were adamant that their practice would not be influenced by these experiences.

GPs found it hard knowing some cancers would be missed because of their decision not to test: for example, one described this as "a burden that I carry" (GP8). GPs most concerned about overdiagnosis experienced the highest levels of personal burden because, although relatively rare, death as the potential consequence of not testing was seen as the worst possible outcome. Some suspected that overdiagnosis and overtreatment were not as much of a burden for the patient as they were for the doctor.

Heuristic 3: GP thinks of each patient as an individual and makes case-by-case decisions

GPs employing heuristic 3 had no preconceived attitude towards avoiding underdiagnosis or overdiagnosis. They tailored PSA testing decisions specifically for the personal circumstances of each patient, according to the patient's risk profile (age, family history), life expectancy, interest, motivation, reason for wanting a PSA test, cancer anxiety, or intention and ability to act on abnormal test results. These GPs were particularly responsive to patients' individual preferences, so the outcome of the consultation was largely unpredictable: "You have to try and work out what's best for the-that one particular patient that you are talking to at the time" (GP18). Testing 'rules' shifted according to the patient and the GP; "it's so easy to just learn what you do from a book but once you are actually faced with someone you know you can't-it's difficult to apply the same rule" (GP6).

These GPs approached communication in several different ways. Some made their own decision about the 'right' approach for each particular patient, and advised that patient accordingly. This could include not discussing overdiagnosis at all, on the grounds that it was irresponsible to expect patients to understand complex information; "if you start going down that road andand to what end?" (GP7). Other GPs tailored their discussion about overdiagnosis to the needs of the individual patient, their perceived level of understanding and time pressures: "it gets more complicated depending on how interested the person is" (GP4). Thus, the GP's communication depended entirely on the individual patient in front of them.

GPs who approached PSA testing case by case generally agreed that overdiagnosis statistics do not, or cannot, apply to individuals; "those like statistical issues don't apply to the individual...because...they make their decisions on a set of complex, but perhaps irrational basis, you know, anxiety and..." (GP7). Accordingly, they tailored their testing and patient communication but expressed some difficulties in translating populationbased information to individuals.

The personal burden experienced by these GPs was minimal as in most consultations the burden of decisionmaking was shared with their patients. GPs sought to reach a mutual understanding of PSA testing if they thought the patient was able to understand the information required, and shared the responsibility of decisions and outcomes of the consultation with the individual man. They tended to consider decisions about PSA testing as neither right nor wrong and so could be swayed either way depending on the patient and their needs. These GPs had minimal legal concern because they perceived patients to have made informed decisions based on their individual needs. 
Heuristic 4: GP preference to avoid thinking about underdiagnosis or overdiagnosis

GPs not thinking about underdiagnosis or overdiagnosis did not have a preference or priority for avoiding one harm over another. For these GPs, the PSA test was considered just another form of routine screening and underdiagnosis or overdiagnosis was not an issue of concern.

The majority of GPs in this group did not engage with considering the implications of underdiagnosis or overdiagnosis and what that meant for their patients. Some of these GPs felt explaining overdiagnosis was the responsibility of urologists, and preferred to simply inform men that a PSA test may lead to them having a biopsy. These GPs also said they preferred to be guided by urologists on what to do about PSA testing overall.

Personal burden associated with underdiagnosis or overdiagnosis was therefore not a significant feature for this group of GPs. For some, their priority was being regarded as a 'good' GP by their patients: they focused on how their testing decisions might influence their reputation and rapport with their patient. They reported that a 'good' GP was in many cases deemed to be someone who actively tested.

\section{DISCUSSION}

Overdiagnosis of indolent cancers in cancer screening is now recognised as a significant problem, but solutions to this problem (eg, communication, public awareness) are as yet uncertain, including in primary care. Most previous research has examined associations between GP characteristics and frequency of PSA testing. Fewer studies have sought to explain variation in GPs' PSA testing practice. Ilic and colleagues ${ }^{46}$ differentiated 'reactive screeners' (GPs who screened only at the patient's request) from 'proactive screeners' (GPs motivated to test, believed screening was beneficial, and feared missing cancer, including for medicolegal reasons). Our study provides a more nuanced analysis of how and why GPs test the way they do, and offers a unique examination of GPs' approaches to prostate cancer underdiagnosis and overdiagnosis. It is the first study to systematically examine the relationship between GPs' reasoning and behaviour in relation to PSA testing. We identified four distinct approaches, each associated with different practices, rationales and outcomes. Our findings explain why men so often receive different advice and clinical care: this depends on their GP's PSA testing practice orientation.

There is value in understanding the reasoning behind actual practice. GPs' reasoning makes sense of variation in practice: it explains why different GPs are making different testing decisions in similar cases. GPs' experiences with PSA testing (positive and negative), values, perceptions (of the GP role, the patient role, of the PSA test and overdiagnosis), considerations of evidence and guidelines, and their sense of personal burden (anticipated or experienced) all uniquely contribute to PSA testing patterns. Variation in practice has ethical implications, as men are experiencing unequal access to information and consent to PSA testing. Yet these GPs were not acting arbitrarily; most were simply doing the best they could in an almost impossible situation. The difficult position GPs are in should be recognised in future efforts to address the problem of prostate cancer overdiagnosis.

\section{Policy implications}

Guidance used by Australian GPs about PSA testing varies widely (see table 1 ). This also contributes to the diversity of practice revealed in this study. Although it would be unrealistic to expect the mere existence of a guideline to change practice ${ }^{39}$ it does seem reasonable for GPs to expect that expert bodies will provide clear guidance wherever possible. A community jury on PSA testing reported men's experiences of variable and inconsistent advice from GPs, and recommended programmes to support GPs to provide patients with better quality and consistent information about PSA screening. ${ }^{47}$ The Australian Medical Health and Research Council (NHMRC) has recently produced an authoritative summary of PSA testing benefits and harms for GPs to discuss with their patients.

The findings of this study offer important guidance for the implementation of such recommendations in practice. We recommend that agencies seeking to promote the uptake of guidance for practitioners must take account of the different motivations of GPs and recognise the significant diversity in the approaches that GPs are taking towards PSA testing of asymptomatic men. GPs who employ heuristic 2, for example, were already attentive to the epidemiological evidence, and so are likely to be receptive to recent NHMRC guidance. However, GPs who are employing heuristic 1 may need very active knowledge translation strategies if they are to change their practice. These GPs were deeply concerned that by their failure to screen they might allow a man to die of prostate cancer. It seems unlikely that they will change their practice unless this concern is recognised and responded to. Communications, workshops and new incentives therefore need to consider variation in GP perspectives and the range of drivers of current practice as identified in this research (address legal concerns, the need for consent due to potential harms and acknowledge burden).

\section{Limitations}

Since physicians with strong opinions may have been more likely to volunteer, some selection bias is possible. However, diverse opinions and approaches were reported, suggesting that strong selection bias is unlikely.

\section{Conclusions}

Future strategies for addressing the problem of prostate cancer overdiagnosis in general practice should be 
underpinned by empirical evidence about how GPs approach PSA testing, and the reasons they give for their actions. Explicit consideration in practice guidelines of the challenges faced by GPs when balancing underdiagnosis and overdiagnosis, including GPs experiences of personal burden, medicolegal concerns and communication strategies, will better support GPs to inform and guide men's decisions on whether or not to have a PSA test. Further public deliberation on how the inevitable trade-offs could and should be managed in primary care could also inform such discussions between clinicians and their patients. ${ }^{48}$

Twitter Follow Kristen Pickles at @PicklesKristen

Acknowledgements The authors thank the general practitioners for their participation in this research.

Contributors KP, SMC and LR conceived the study and were involved in designing the study, developing the methods and drafting of the manuscript. SMC and LR obtained funding and are Cls on the NHMRC funded project grant. KP conducted the interviews, had full access to all data in the study, and takes responsibility for the integrity of the data and the accuracy of the data analysis. All authors contributed to the interpretation of the analysis and critically revised the manuscript.

Funding The project was funded by NHMRC grant 1023197. Stacy Carter is supported by NHMRC Career Development Fellowship 1032963. The funders had no role in the design or conduct of the study; in the collection, analysis and interpretation of the data; or in the preparation or approval of the manuscript.

Competing interests SC is supported by NHMRC Career Development Fellowship 1032963

Ethics approval All study procedures were approved by the Cancer Institute NSW and the University of Sydney Human Research Ethics Committee [\#15245].

Provenance and peer review Not commissioned; externally peer reviewed.

Data sharing statement No additional data are available.

Open Access This is an Open Access article distributed in accordance with the Creative Commons Attribution Non Commercial (CC BY-NC 4.0) license, which permits others to distribute, remix, adapt, build upon this work noncommercially, and license their derivative works on different terms, provided the original work is properly cited and the use is non-commercial. See: http:// creativecommons.org/licenses/by-nc/4.0/

\section{REFERENCES}

1. Stark JR, Mucci L, Rothman KJ, et al. Screening for prostate cancer remains controversial. BMJ 2009;339:b3601.

2. Ilic D, Neuberger MM, Djulbegovic M, et al. Screening for prostate cancer. Cochrane Database Syst Rev 2013:1-76.

3. Moyer VA. Screening for prostate cancer: US. preventive services task force recommendation statement. Ann Intern Med 2012:157:120-34.

4. Djulbegovic M, Beyth RJ, Neuberger MM, et al. Screening for prostate cancer: systematic review and meta-analysis of randomised controlled trials. BMJ 2010;341:c4543.

5. Mackie A. Screening for Prostate Cancer Review against programme appraisal criteria for the UK National Screening Committee (UK NSC). London: National Health Service, 2010.

6. Royal Australian College of GPs (RACGP). Guidelines for preventive activities in general practice 2012. 8th edn. http://www.racgp.org.au/ your-practice/guidelines/redbook/early-detection-of-cancers/prostatecancer/

7. Wilt TJ, Ahmed HU. Prostate cancer screening and the management of clinically localized disease. BMJ 2013;346:f325.

8. Carter HB, Albertsen PC, Barry MJ, et al. Early detection of prostate cancer: AUA guideline. J Urol 2013;190:419-26.
9. Mitka M. Group now advises against routine PSA screening. JAMA 2013;309:2316.

10. American Cancer Society (ACS). American Cancer Society recommendations for the early detection of prostate cancer 2014 http://www.cancer.org/cancer/prostatecancer/moreinformation/ prostatecancerearlydetection/prostate-cancer-early-detection-acsrecommendations

11. National Health and Medical Research Council (NHMRC). Australia. PSA testing for prostate cancer in asymptomatic men: Information for health practitioners. 2014; https://www.nhmrc.gov.au/guidelinespublications/men4, 2014.

12. National Health Service (NHS) Choice, United Kingdom. Prostate cancer - PSA screening. 2015; http://www.nhs.uk/Conditions/ Cancer-of-the-prostate/Pages/prevention.aspx

13. Cancer Council Australia, Australian Health Ministers' Advisory Council (AHMAC), Australia. Position statement. Prostate cancer screening in Australia: joint key messages. 2010; http://www.cancer. org.au/content/pdf/CancerControlPolicy/PositionStatements/PSProstate_Cancer_Screening_Joint_key_messages_\% 20published_May2010.pdf.

14. Urological Society of Australia and New Zealand (USANZ). Urological Society of Australia and New Zealand PSA testing policy. 2009; http://www.usanz.org.au/uploads/65337/ufiles/PDF/psatesting.pdf

15. Qaseem A, Barry MJ, Denberg TD, et al. Screening for prostate cancer: a guidance statement from the Clinical Guidelines Committee of the American College of Physicians. Ann Intern Med 2013;158:761-9.

16. Ross LE, Coates RJ, Breen N, et al. Prostate-specific antigen test use reported in the 2000 National Health Interview Survey. Prev Med 2004;38:732-44.

17. Sirovich BE, Schwartz LM, Woloshin S. Screening men for prostate and colorectal cancer in the United States: does practice reflect the evidence? JAMA 2003:289:1414-20.

18. Australian Institute of Health and Welfare (AIHW). Prostate cancer in Australia. 2013; http://www.aihw.gov.au/WorkArea/DownloadAsset. aspx?id=60129545133

19. Welch G, Schwartz L, Woloshin S. Overdiagnosed: making people sick in the pursuit of health. Beacon Press, 2011.

20. Hersch J, Jansen J, Irwig L, et al. How do we achieve informed choice for women considering breast screening? Prev Med 2011:53:144-6.

21. Etzioni R, Penson DF, Legler JM, et al. Overdiagnosis due to prostate-specific antigen screening: lessons from U.S. prostate cancer incidence trends. J Natl Cancer Inst 2002;94: 981-90.

22. Draisma G, Boer R, Otto SJ, et al. Lead times and overdetection due to prostate-specific antigen screening: estimates from the European Randomized Study of Screening for Prostate Cancer. J Natl Cancer Inst 2003:95:868-78.

23. McGregor M, Hanley J, Boivin J, et al. Screening for prostate cancer: estimating the magnitude of overdetection. CMAJ 1998;159:1368-72.

24. Telesca D, Etzioni R, Gulati R. Estimating lead time and overdiagnosis associated with PSA screening from prostate cancer incidence trends. Biometrics 2008;64:10-19.

25. Gulati R, Wever EM, Tsodikov A, et al. What if I don't treat my PSA-detected prostate cancer? Answers from three natural history models. Cancer Epidemiol Biomarkers Prev 2011;20:740-50.

26. Wever EM, Draisma G, Heijnsdijk EA, et al. Prostate-specific antigen screening in the United States vs in the European Randomized Study of Screening for Prostate Cancer-Rotterdam. J Natl Cancer Inst 2010;102:352-5.

27. Moynihan R, Doust J, Henry D. Preventing overdiagnosis: how to stop harming the healthy. BMJ 2012;344:e3502.

28. Cooperberg MR, Broering JM, Kantoff PW, et al. Contemporary trends in low risk prostate cancer: risk assessment and treatment. J Urol 2007:178:S14-19.

29. Welch HG, Black WC. Overdiagnosis in cancer. J Natl Canc Inst 2010;102:605-13.

30. Sanda MG, Dunn RL, Michalski J, et al. Quality of life and satisfaction with outcome among prostate-cancer survivors. $N$ Engl $J$ Med 2008;358:1250-61.

31. Hayes $\mathrm{JH}$, Ollendorf DA, Pearson SD, et al. Active surveillance compared with initial treatment for men with low-risk prostate cancer: a decision analysis. JAMA 2010;304:2373-80.

32. Loeb S, Catalona WJ. What to do with an abnormal PSA test. Oncologist 2008;13:299-305

33. Aus G, Bergdahl S, Lodding P, et al. Prostate cancer screening decreases the absolute risk of being diagnosed with advanced 
prostate cancer-results from a prospective, population-based randomized controlled trial. Eur Urol 2007;51:659-64.

34. Grubb RL, Pinsky PF, Greenlee RT, et al. Prostate cancer screening in the Prostate, Lung, Colorectal and Ovarian cancer screening trial: update on findings from the initial four rounds of screening in a randomized trial. BJU Int 2008;102:1524-30.

35. Schröder FH, Hugosson J, Carlsson S, et al. Screening for prostate cancer decreases the risk of developing metastatic disease: findings from the European Randomized Study of Screening for Prostate Cancer (ERSPC). Eur Urol 2012;62:745-52.

36. Cooper CP, Merritt TL, Ross LE, et al. To screen or not to screen, when clinical guidelines disagree: primary care physicians' use of the PSA test. Prev Med 2004;38:182-91.

37. Brett J, Watson E, Hewitson P, et al. PSA testing for prostate cancer: an online survey of the views and reported practice of general practitioners in the UK. BMC Fam Pract 2005;6:24.

38. Gormley GJ, Catney D, McCall JR, et al. Prostate-specific antigen testing: uncovering primary care influences. BJU Int 2006;98:996-1000.

39. Girgis S. General practitioners' perceptions of medicolegal riskusing case scenarios to assess the potential impact of prostate cancer. Med J Aust 1999;171:362-6.

40. Pollack CE, Platz EA, Bhavsar NA, et al. Primary care providers' perspectives on discontinuing prostate cancer screening. Cancer 2012;118:5518-24.
41. Drummond FJ, Carsin A, Sharp L, et al. Factors prompting PSA-testing of asymptomatic men in a country with no guidelines: a national survey of general practitioners. $B M C$ Fam Pract 2009;10:3.

42. Bell DS, Hays RD, Hoffman JR, et al. A test of knowledge about prostate cancer screening. J Gen Intern Med 2006;21:310-14.

43. Clements A, Watson E, Rai T, et al. The PSA testing dilemma: GP's reports of consultations with asymptomatic men: a qualitative study. BMC Fam Pract 2007;8:35.

44. Bowen DJ, Hannon PA, Harris JR, et al. Prostate cancer screening and informed decision-making: provider and patient perspectives. Prostate Cancer Prostatic Dis 2011;14:155-61.

45. Charmaz K. Constructing grounded theory: a practical guide through qualitative analysis. London: SAGE Publications, 2006.

46. Ilic D, Murphy K, Green S. What do general practitioners think and do about prostate cancer screening in Australia? Aus Fam Phys 2013;42:904-8.

47. Rychetnik L, Doust J, Thomas R, et al. A Community Jury on PSA screening: what do well-informed men want the government to do about prostate cancer screening-a qualitative analysis. BMJ Open 2014:4:e004682.

48. Rychetnik L, Carter SM, Abelson J, et al. Enhancing citizen engagement in cancer screening through deliberative democracy. J Natl Canc Inst 2013;105:380-6. 